\title{
PROTOCOLIZACIÓN DE PROCEDIMIENTOS INSTITUCIONALES, INVESTIGACIÓN EVALUATIVA Y CUMPLIMIENTO DE LOS DERECHOS DE LAS PERSONAS MENORES DE EDAD MIGRANTES NO ACOMPAÑADAS EN COSTA RICA
}

\section{PROTOCOLIZATION OF INSTITUTIONAL PROCEDURES, EVALUATION RESEARCH AND FULFILLMENT OF THE RIGHTS OF UNACCOMPANIED MIGRANT MINORS IN COSTA RICA}

\section{Carlos Yurán Chavarría Carranza*}

RESUMEN

La investigación evaluativa es un instrumento de suma utilidad para conocer aquellos momentos e instancias donde las organizaciones internamente y en su relación con otras, puedan conocer las debilidades y fortalezas que enfrentan en el cumplimiento de sus objetivos. Una situación como la de las personas menores de edad migrantes no acompañadas, motivó a la Organización Internacional de las Migraciones a realizar una evaluación sobre la respuesta societaria que se da en Costa Rica a la atención de tal problemática. Los resultados de esta indagación muestran importantes aciertos respecto a la respuesta societaria la cual se clasifica como eficiente. También se muestran aquellos otros en los cuales este accionar interno y coordinado entre las instituciones, fragiliza y hasta tergiversa el cumplimiento de los Derechos Humanos que abrigan a las poblaciones menores de edad en el país.

PALABRAS CLAVE: INVESTIGACIÓN * EVALUACIÓN * MIGRACIÓN * NIÑO * DERECHOS HUMANOS

\section{ABSTRACT}

Evaluation research is a very useful tool in those instances where organizations, internally and in their relations with others, can identify their weaknesses and strengths with which they accomplish their objectives. A situation such as the migration of unaccompanied minors made the International Organization for Migration develop an assessment of the societal response given in Costa Rica to attend to the problem. The results of this investigation show significant successes societal response is considered efficient. However, they also reveal moments where the internal and coordinated actions among institutions, weaken and even distort the fulfillment of Human Rights which should protect minors in the country.

KEYWORDS: RESEARCH * EVALUATION * MIGRATION * CHILDREN * HUMAN RIGHTS

Sede Occidente de la Universidad de Costa Rica.

carlos.chavarria@ucr.ac.cr 


\section{INTRODUCCIÓN}

En el presente artículo se pretende aportar evidencia sobre la importancia de que los procesos de trabajo tendientes al abordaje de situaciones relacionadas con personas menores de edad se encuentren debidamente protocolizados; no solo porque es un recurso fundamental para dirigir toda labor organizacional, sino porque de ello se desprende, a su vez, la posibilidad instrumental de identificar aquellas acciones (o falta de estas) y lugares donde se tergiversa $y / 0$ distorsiona el cumplimiento de los objetivos organizacionales, principalmente, en cuanto a situaciones tan sensibles como el resguardo de los Derechos Humanos de las personas menores de edad.

El material sobre el que se realiza tal análisis se desprende del trabajo titulado "Situación de niños, niñas y adolescentes que viajan no acompañados por la Región Centroamericana" (Chavarría y Robert, 2011) para la Organización Internacional de las Migraciones (огм). Si bien, en el citado trabajo se aborda la respuesta societaria (Rs) que se brinda en Costa Rica a la problemática y a la sensible situación que viven las personas menores de edad migrantes no acompañadas (рммла) en nuestra región y a nivel mundial, el interés en el presente documento se concentra en mostrar la forma en que un proceso de investigación evaluativa permite establecer las debilidades que muestra una organización en su intento por cumplir con sus objetivos, que al final son su razón de ser.

IMPORTANCIA DE LA PROTOCOLIZACIÓN DE PROCEDIMIENTOS PARA EL LOGRO DE LOS OBJETIVOS ORGANIZACIONALES

Como bien señaló el psicólogo social Ignacio Martín-Baró (1989), una organización es un sistema social configurado para lograr ciertos objetivos, pueden ser económicos como en una empresa mercantil o servicios de calidad como en un ministerio o una organización religiosa. Para tener éxito en su cometido, las personas que conforman la organización deben compartir un mínimo de conocimientos, valores $y$ fines.
No se puede en este texto debatir sobre los aspectos que demandan las organizaciones modernas para su buen funcionamiento, ello es tema de un debate continuo en las ciencias sociales (Chavarría, 2014; Cruz, López y Ramírez, 2007; González, 1996). No obstante, lo que se hace necesario es resaltar la importancia que cobra la revisión constante del desempeño organizacional como forma de mejorar un proceder que naturalmente propende a la entropía (Chavarría, 2014) o a la desorganización.

Peter Senge (1992) afirmaba que una organización inteligente es la que aprende con el fin de ampliar su futuro. Dentro de otros aspectos, regularmente se afirma que el futuro de una organización se encuentra inevitablemente asociado a la satisfacción del cliente, llámese este público meta o simplemente al logro de los objetivos. Es común escuchar referirse a esto como "brindar servicios de calidad" (Chang, 2005; González, 1996; González, Moreno y Peris, 2001; Senile, 1996; Silverman, 1992; Singh, 1999; Wellington, 1995).

Una condición central para brindar un servicio de calidad es que se encuentre bien definido, dispuesto, sea accesible, sea brindado a tiempo, entre otros aspectos (Wellington, 1995), es decir, que esté protocolizado. En ese sentido, un protocolo es una guía de actividad desarrollada para describir y guiar el quehacer organizacional con el fin de ofrecer un servicio o producto de calidad. En la medida en que el desempeño organizacional se encuentre protocolizado, y que esos protocolos estén abiertos a ser modificados por vía del aprendizaje que se da principalmente en las personas que los ejecutan, es que se habla de organizaciones inteligentes (Cruz, López y Ramírez, 2007; Senge, 1992).

\section{PERTINENCIA DE LA INVESTIGACIÓN EVALUATIVA PARA EL MEJORAMIENTO ORGANIZACIONAL}

La necesidad de investigación y de producción de información está dada por una base justificatoria, es decir, por la descripción de las diversas problemáticas que debe enfrentar toda organización a cada momento (Chavarría, 2014) 
y que se acusan como necesarias de ser comprendidas (Gallardo, 1991).

En el caso de una organización, problemas como quejas de clientes, ineficiencia en el cumplimiento de objetivos, entrabamiento de procesos, elevación en los costos de operación, aumento de accidentes y enfermedades ocupacionales, entre otros, son abordados por investigaciones denominadas evaluativas.

No importa si el alcance de la información producida de este tipo de investigaciones es descriptiva o explicativa (Venegas, 1999), lo relevante es que se busca, en el plano descriptivo establecer el desempeño y/o los resultados de un programa y el curso de acción de una organización; $y$ en el plano explicativo determinar la relación de tal comportamiento con el grado en que se logran los objetivos propuestos (Venegas, 1999).

La investigación evaluativa se decanta como prioritaria y cada vez cumple un papel más significativo para aquellas organizaciones inteligentes (Senge, 1992) que entienden que su éxito y permanencia en el tiempo dependen en gran medida de atender las distintas desviaciones a las que propenden internamente de forma natural $y$ por presiones del ambiente (Chavarría, 2014).

A partir del año 1954, en el que se promulgó la denominada Norma Jis z 9022 en Japón, estableciendo el Método del Diagrama de Control (figura 1), se superaron las ideas administrativas clásicas. La fase mecánica (prevenir, planear y organizar) y dinámica (dirigir y controlar), se verían desplazadas por un modelo circular que inicia con la Planeación, luego la Ejecución, posteriormente la Evaluación y por último, la Corrección.

Todo ello de un modo continuo para dirigir el cambio necesario, en consideración de que tal condición debe ser vista como una oportunidad para establecer las acciones correctivas que lleven a mejorar el desempeño organizacional, ya que toda evaluación tiene siempre como visión perfectibilidad de la organización (González, 1996; Chavarría, 2014).

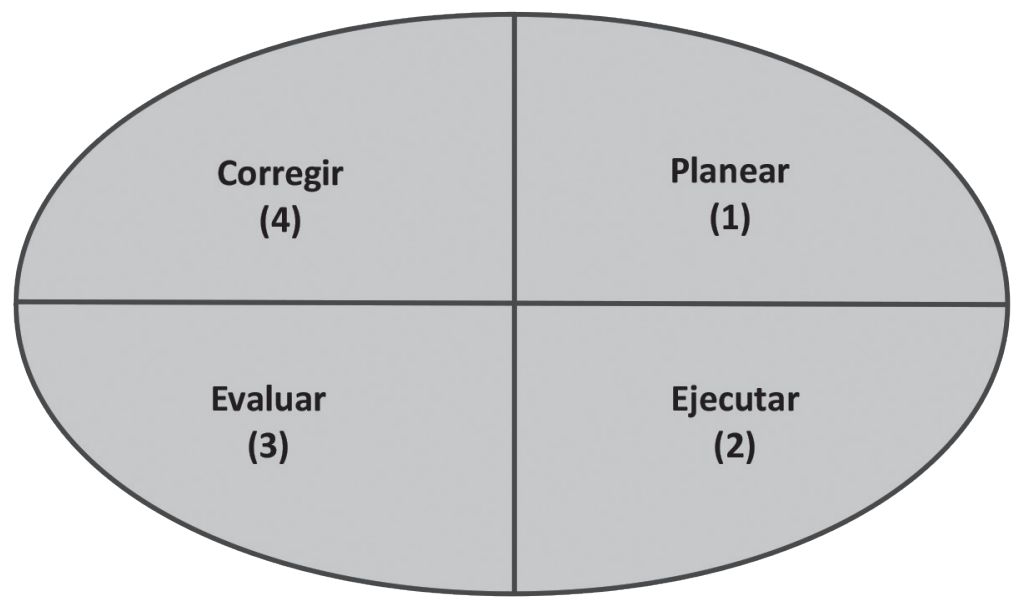

Fuente: Shewhart, W. citado en González, 1996.

RESPUESTA SOCIETARIA COSTARRICENSE A LA SITUACIÓN DE PMMNA

Dado que las pмmna son por definición migrantes irregulares, ya que no cuentan con ninguna persona mayor responsable que los tutele y cuide, es que generalmente se mantienen en la clandestinidad hasta que son contactadas por funcionarios o funcionarias de alguna organización en el país. Desde este momento se activan las acciones que, protocolizadas o no, 
se despliegan en un conjunto de organizaciones para atender la situación hasta que se llegue a una solución definitiva.

La articulación que se da entre políticas, leyes, normativas, instituciones, coordinaciones y prácticas organizacionales con el fin de dar atención y resguardar a las pмMNA — quienes son por antonomasia, personas en alto grado de vulnerabilidad- es lo que se define en este caso como respuesta societaria (Rs) (Chavarría $y$ Robert, 2011).

Las características de la problemática hacen que intervengan instituciones nacionales de diversa índole, incluidas organizaciones no gubernamentales, que en conjunto conforman la rs del país. También cuentan en esta Rs los consulados y embajadas extranjeras acreditadas en el país y que son parte central en todo el proceso, sobre todo en la parte de identificación de la pмmna $y$ de las coordinaciones para su repatriación (si este fuera el caso).

Desde que una рммnа es contactada $y$ queda a resguardo de alguna organización, hasta que es repatriada a su país de origen, o en su defecto cuando queda bajo algún recurso de acogimiento a nivel nacional y se regulariza su estatus migratorio, son múltiples las acciones o subprocesos que se despliegan en toda la red societaria.

Es importante resaltar que el conjunto de acciones que se realizan no están recogidas en un único protocolo que describa paso a paso lo que debe hacerse en cada organización y/o en la coordinación entre ellas. Pero entonces, ¿cómo evaluar la rs en ausencia de este indispensable recurso?

Para que esto fuera posible se hizo necesaria la tarea de producir un protocolo completo a partir de otros estatuidos sobre acciones parciales que tienen que ver o son semejantes a la problemática de PMмNa, solo así se podrían identificar las falencias y aciertos intra e interinstitucionales, $y$ en consecuencia como país, al momento de atender estos casos. La construcción de ese protocolo conjuntado se logró de la siguiente manera:

1. Establecer el marco legal a que se obliga el país en materia de personas menores de edad en términos generales $y$ en condición de migrante no acompañadas en particular. Entre ellos destacan (Chavarría y Robert, 2011):

$\diamond \quad$ La Declaración Universal de los Derechos Humanos y sus protocolos (1948).

$\diamond \quad$ La Convención Americana de Derechos Humanos (1969).

$\diamond \quad$ Ley 7184 Ratificación de la Convención Sobre los Derechos del Niño (1990).

$\diamond \quad$ Ley 7739 Código de la Niñez y la Adolescencia (1997).

$\diamond \quad$ Ley 8032 Aprobación de la Convención Latinoamericana Sobre Restitución Internacional de Menores (2000).

$\diamond \quad$ Ley 8315 Protocolo Para Prevenir, Reprimir y Sancionar la Trata de Personas, Especialmente Mujeres y Niños (2002).

$\diamond \quad$ Ley 8487 Ley de Migración y Extranjería (2005).

También de esta manera se establecieron los derechos que resguardan la condición de vulnerabilidad que viven las PMMNA y que, eventualmente, podrían ser tergiversados o no atendidos, entre los que destacan (Carazo y Salas, 2003):

$\diamond \quad$ Derecho a la vida.

$\diamond \quad$ Derecho a la igualdad $y$ a la no discriminación.

$\diamond \quad$ Derecho a una vida digna.

$\diamond \quad$ Derecho a la educación.

$\diamond \quad$ Derecho a la salud.

$\diamond \quad$ Derecho al trabajo.

$\diamond \quad$ Derecho a la identidad.

$\diamond \quad$ Derecho a la familia.

$\diamond \quad$ Derecho a la integridad $y$ libertad.

$\diamond \quad$ Derecho a la libre movilización.

2. Acopiar los diversos protocolos de actividad de distintas organizaciones que deben atender situaciones que involucren a personas menores de edad. También de 
aquellas que, aunque no tienen a esta población por objeto directo, la toman en cuenta por su condición de persona migrante. Tal es el caso, por ejemplo, de la Dirección de Migración y Extranjería (DGME). Los principales protocolos considerados fueron (Chavarría y Robert, 2011):

$\diamond \quad$ Conferencia Regional sobre Migraciones (2009). Lineamientos regionales para la atención de niños, niñas $y$ adolescentes migrantes no acompañados en casos de repatriación. En dicho trabajo se describen los compromisos de los Estados miembros de este grupo, al que pertenece Costa Rica, sobre las acciones que deben seguir para garantizar la protección integral de personas menores de edad migrantes cuando son repatriadas.

$\diamond \quad$ OIM-UNicEF (2007). En el marco de los compromisos señalados en el documento anterior $y$ de los derechos humanos que protege a las personas menores de edad víctimas de alguna modalidad de trata cuando vayan a ser repatriadas, se especifican en este documento las acciones concretas que deben seguirse en todos los momentos que involucran este procedimiento.

$\diamond \quad$ PANI (2009). Esta es la página electrónica del Patronato Nacional de la Infancia, donde se expone la ley constitutiva ratificada en 1990 y se le define como la institución rectora a nivel nacional de la materia según el Código de la Niñez y la Adolescencia (Ley 7739) de 1977 y su Ley Orgánica (Ley 7648) de 1996. Ambas leyes se constituyen en el marco legal mínimo para la protección de los derechos de la niñez y la adolescencia en Costa Rica $y$ definen las responsabilidades del PANi en la materia. $\diamond \quad$ PANI-OIM (2007). Este documento se enfoca en el tratamiento nacional que se debe dar al tema de la "trata de personas menores de edad" y el marco legal, que a nivel nacional e internacional define el encuadre general de actividad donde se circunscriben las distintas acciones a emprender para la atención integral de la problemática en el Estado costarricense.

De la revisión de estos documentos se pudieron extraer el conjunto de acciones estipuladas para atender la problemática de las рммла. Las acciones fueron agrupadas en cuatro diferentes momentos dado que tienen objetivos e involucran actores distintos: momento de detección, momento de introducción al sistema, momento de institucionalización y momento de repatriación.

Valga reseñar que, como recomendación en el trabajo de marras (Chavarría y Robert, 2011), se propuso que el protocolo construido fuera considerado como base para ser revisado y corregido de tal manera que termine por constituirse en el protocolo oficial del país.

\section{MÉTODO}

El estudio base de este reporte se realizó mediante una investigación evaluativa de tipo formativo. Al respecto se señala:

En general, la evaluación formativa se aplica en la fase de ejecución de un programa, proyecto $y$ es especialmente recomendable cuando este tiene etapas claramente definidas, con metas intermedias y a largo plazo, o en proyectos que admiten, a manera de retroalimentación, una modificación en la marcha (Correa, Puerta y Restrepo, 2002).

Con esta investigación, se pretendió conocer si la respuesta organizada (Rs) corresponde con las necesidades que experimentan en el tránsito las рммма, la prevención de los riesgos y el acompañamiento hasta su instalación en una red de apoyo social $y / 0$ societario que garantice 
condiciones aceptables para su desarrollo integral y el resguardo de los derechos humanos.

\section{PARTICIPANTES}

Para conocer la rs con la mayor prolijidad posible, en el marco de los límites temporales dispuestos para la investigación, se generó un tipo de muestreo denominado en cadena o en red (Hernández, Fernández y Baptista, 2003). Este tipo de muestra propicia lo que se conoce como el efecto bola de nieve, que se refiere a la posibilidad de que a partir de la identificación $y$ contacto inicial con entidades $y$ su personal, $y$ merced a la información que brindan, se identifican y contactan otras organizaciones, funcionarios $y$ funcionarias que pueden incluirse en el estudio para enriquecer la exploración y evaluación. Esta práctica se sigue hasta que la variación en la información generada resulta mínima o irrelevante. En el cuadro 1, se presenta el total del personal que fue considerado mediante este mecanismo, la organización a la que pertenece y la ocupación que ejerce.

\section{CUADRO 1}

FUNCIONARIOS Y FUNCIONARIAS QUE DIERON ENTREVISTAS, INSTITUCIÓN A LA QUE PERTENECEN Y ROL EN LA ATENCIÓN DEL FENÓMENO MIGRATORIO DE PMMNA JULIO-NOVIEMBRE, 2009

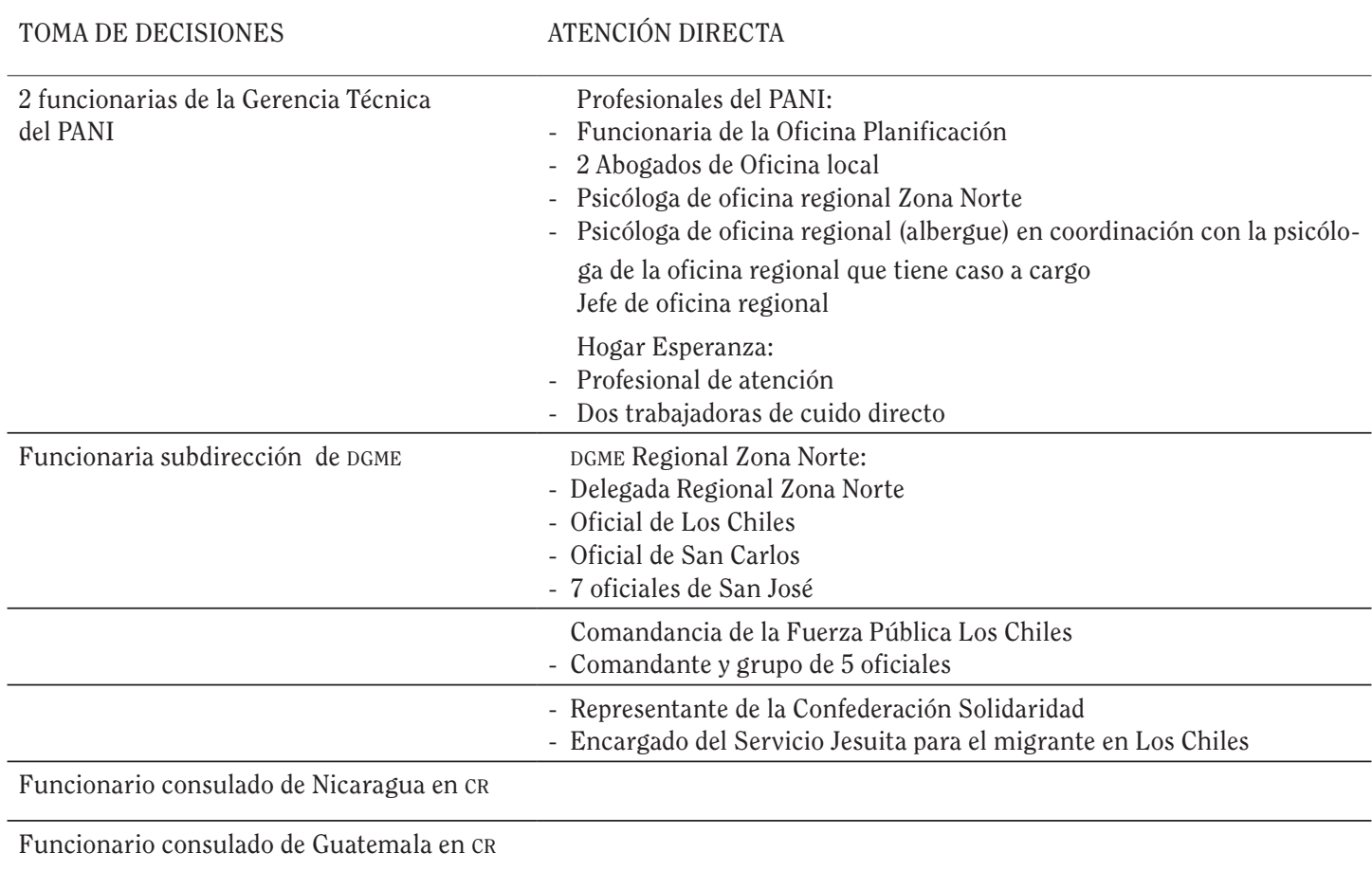

Fuente: Elaboración propia a través del trabajo de campo.

\section{INSTRUMENTOS}

En apego a todas las consideraciones de tipo legal $y$ formas de actuación descritas y conjuntadas en el Protocolo Integrado sobre la Rs a la problemática de las PMmNa y al considerar los momentos de acción en que cada entidad debe actuar, se construyó una serie de cuestionarios que variaban entre sí en cuanto a los aspectos particulares que debían establecerse según la naturaleza de cada organización. 
Cada cuestionario fue aplicado de forma directa por el equipo de investigación a la persona o de forma grupal, según fuera el caso. El conjunto de estos instrumentos consta en el informe completo de la investigación (Chavarría y Robert, 2011).

\section{RESULTADOS}

El detalle sobre el recorrido realizado al conjunto de instituciones que interviene de alguna forma para atender la situación de una Pмmna aparece en el informe de Chavarría y Robert (2011). También, se puede consultar el protocolo unificado que se produjo con el fin de hacer viable la tarea evaluativa. Por otra parte, valga aclarar que el hecho de que en este artículo se concentre en exponer las debilidades de esta Rs se debe tanto a razones de espacio como al objeto de trabajo que se propuso reportar. Son muchos los aciertos, fortalezas y logros institucionales que en esta materia se evidencian en el país.

No obstante, esta exposición se centra en detectar aquellos momentos y lugares donde la rs costarricense se muestra débil o desarticulada para atender la problemática de рммла, con lo cual se puede tergiversar $y / 0$ incumplir con la legalidad que rige la materia de derechos que protegen a estas personas. Con el fin de diferenciar cada debilidad se reportarán de forma numerada.

1. No existe un protocolo específico para la atención nacional de la problemática de рммnа. En estos casos, generalmente, se siguen algunos procedimientos del "Protocolo para la trata de personas menores de edad", elaborado por el PANI en el 2007, y que procura responder operativamente a la obligación que tiene el Estado costarricense, firmante de la Convención sobre los Derechos del Niño (CDN-1989-1990), de adoptar las medidas administrativas, legislativas, presupuestarias y de cualquier índole, que garanticen la plena efectividad de los derechos fundamentales de las personas menores de edad, en particular el derecho a la vida (art. 12), a la protección estatal (art. 13), a la libertad (art. 14), al libre tránsito (art. 15), a la protección ante el peligro (art. 19), y a la información (art. 20).

2. Una vez que la pmmna es introducida al sistema, está el Pani en el centro de las coordinaciones, no obstante, no en todos los casos resultan fluidas, efectivas y articuladas con esta institución. No en pocos casos, sobre todo con menores de edad de países fronterizos, funcionarios $y$ funcionarias de la DGME repatrian PммNA sin coordinar con el Pani, contraviniéndose así los compromisos que el país ha contraído sobre protección temporal y repatriación voluntaria. En otros casos, las coordinaciones no son posibles porque alguna de las entidades, incluido el PANI, no tiene a disposición los recursos para dar una respuesta oportuna $o$ incluso, porque se considera que el caso no es de su competencia.

3. Asociado también a los problemas de coordinación interinstitucional, se encuentra el hecho de que los procedimientos seguidos por las diferentes organizaciones para atender a las pmmn desde que son detectadas hasta que se alcanza a definir una situación más estable para ellas, hace que se implique un número amplio de funcionarios $y$ funcionarias, lo que no es conveniente para una persona menor en estado de vulnerabilidad.

4. La razón de las fallas de coordinación interinstitucional, ya sean estructurales, funcionales, actitudinales o de escasez de recursos, conllevan a incurrir en una violación del art. 17 del Código de la Niñez y la Adolescencia (cDN) sobre la libre movilización.

5. Estos problemas de coordinación trascienden las fronteras de la cultura institucional nacional, alcanzando a las relaciones con los consulados y entidades similares al PANI en los países de origen de las pmmna. Problemas de coordinación 
interinstitucional internacional que inciden en dificultades de identificación, evaluación y toma de decisiones, con frecuencia, conllevan a la indefinición jurídica de la pmmna, así como, al menoscabo de derechos básicos y al inadecuado acceso a servicios básicos como los de salud, educación y demás programas en beneficio de las personas menores de edad que se implementan en los albergues del país. Cuanto mayor sea el tiempo que la Pмmna se encuentre en esa situación, más acusado será el incumplimiento de su derecho a tener una identidad (art. 8, cDN y art. 23, cNA) y de incorporarse plenamente a los programas y servicios existentes en su beneficio.

6. La situación de indefinición antes descrita no debiera ser obstáculo para que las рммма sean admitidas $y$ atendidas normalmente en los centros de salud $y$ de educación, dado que existen convenios firmados por el Pani con las instituciones responsables para que no se le niegue el acceso a los servicios a ninguna persona menor de edad, no obstante, en algunos casos debido el desconocimiento de los funcionarios $y$ las funcionarias sobre el detalle funcional de estos convenios 0 por la ausencia de un protocolo preciso de su implementación, se determina la existencia práctica de diversos obstáculos que atentan contra el interés superior de la persona menor.

7. Otra debilidad relacionada con la coordinación interinstitucional internacional se da como producto del desconocimiento que parece existir sobre algunos recursos $y$ apoyos que brindan organismos internacionales como la oIm, el Servicio Social Internacional o la Conferencia Regional sobre Migración (сRм), principalmente en facilitar procesos de comunicación entre instituciones de países, aún aquellos donde no se cuenta con representación consular o con fondos especiales para procesos de repatriación.
8. No existe ningún control centralizado en el Pani, en tanto institución responsable de velar por el interés superior de la persona menor de edad en general y de la pммna en particular, sobre la atención de estos casos en el país. En consecuencia no se cuenta con un panorama y seguimiento confiables de la dinámica ni del abordaje societario en términos generales de esta problemática.

En la oficina de Planificación del Pani se afirma que la práctica de aportar los datos referidos al fenómeno de los $y$ las menores migrantes para realizar análisis estadísticos se abandonó hace algún tiempo, lo cual confirma este reporte. Los registros actuales toman como fundamento el tipo de situación que fue atendida, sea esta maltrato, deambulación, explotación sexual, trata de persona, conflicto con la ley, entre otros, sin especificar si la persona atendida es migrante o no. Por ello, determinar las particularidades que rodean al fenómeno migratorio como lugar de procedencia, forma de detección, acciones emprendidas, entre otras, son imposibles de establecer mediante estos registros.

9. Tampoco se efectúa ningún tipo de registro sistemático al respecto en ninguna de las otras organizaciones consultadas.

10. La información de los expedientes de las pмmNa en el pani adolece de falta de clasificación, sistematicidad, consistencia $y$ actualización, por lo que se dificulta localizar, evaluar y dar seguimiento a los casos.

11. En la dgme los casos de pmmna que son entregados al PANI no se registran en expedientes. Al parecer lo que existe es un archivo general donde se acumulan los informes de formato inespecífico que los funcionarios y las funcionarias realizan para cada caso. 
12. No se realizan acciones tendientes a regularizar la permanencia legal de la рммNa en el país, mediante la definición de algún estatus como por ejemplo, el refugio o algún otro estatus temporal. En el informe completo de esta indagación (Chavarría y Robert, 2011) se señaló que en el momento de la institucionalización se debía "valorar la posible condición de refugio" como forma de dar algún estatus migratorio y legalizar a la persona menor en el país. Además, se afirmó que esta tarea corresponde a la DGME; sin embargo, funcionarios y funcionarias de esta institución aseguran que el Pani debe representar a la persona menor frente a la DGME para realizar esta solicitud. Se estableció que en ninguno de los casos conocidos se llevó adelante este procedimiento.

13. La información brindada por las pмmNa con frecuencia es poco fiable, como resultado de sus expectativas, circunstancias migratorias y estrategias de sobrevivencia, lo que dificulta la coordinación con consulados y entidades homólogas de los otros países. Se suman a esto las limitaciones de coordinación mencionadas. Todo en conjunto dificulta la incorporación de las PммNa a los programas y servicios que se implementan en su beneficio e interés superior.

14. La evaluación de la situación de la pmmna y la determinación del curso de acción más conveniente a sus intereses - sea la repatriación o el otorgamiento de la condición de refugio- parece depender en mayor medida del interés por el caso $y$ por la coordinación lograda con los consulados $y$ entidades responsables en los países de origen, en vez del estricto cumplimiento de la normativa establecida en los convenios. Se determinó que cada país tiene sus propias condiciones para cumplir con estos requisitos y condiciones estipuladas. En las entrevistas realizadas se señaló como ejemplo, que cuando se ha solicitado alguna documentación que lleve a establecer la identidad de la persona menor de edad o en alguna otra acción, algunos países suramericanos son más ágiles en ello, frente a un país cercano como Nicaragua, en el cual algunos casos se han prolongado en el tiempo debido a que las condiciones de registro y emisión de documentos no cuenta con mayor desarrollo tecnológico, por lo que acceder a ellos es más difícil.

15. Los albergues del pani o aquellos administrados por ongs, no reúnen las condiciones apropiadas para dar una respuesta que garantice la salvaguarda de los derechos e intereses superiores de las рммNa en diversas situaciones; por ejemplo, cuando están solo de tránsito, son víctimas de alguna modalidad de trata de personas, son utilizadas para el tráfico de sustancias o no quieren ser repatriados por alguna razón.

16. Lo anterior es particularmente sensible cuando una persona menor migrante que viaja como parte de un grupo con familiares adultos, es separada y puesta bajo la responsabilidad del pani. En el momento de realizar las entrevistas a los funcionarios y las funcionarias de las distintas instituciones, era reciente el caso de unos grupos de migrantes detectados a mediados del año 2009, que venían procedentes de África, sobre todo de Somalia, y llevaban rumbo a los Estados Unidos (Madrigal, 2009). Aunque algunas de las personas menores de edad viajaban sin otros familiares, las que si lo hacían fueron separadas de su grupo familiar una vez que fueron puestas a resguardo en los albergues del PANI. Esto generó la situación paradójica de que, una vez las personas adultas fueron expulsadas del país, se dejó a las personas menores en condición de PMMNA, con lo que se violaron los art. 8 y 9 de la Convención de los Derechos del Niño y la Niña que estipulan el derecho de todo 
menor a mantenerse como parte de su grupo familiar o primario de apoyo.

17. Para el caso de las pmmna que provienen de países con culturas lingüísticas $y$ tradiciones muy distintas (casos reseñados como: Pmmna de Somalia, Eritrea, Rumanía, entre otros) no se cuenta con el personal ni las condiciones idóneas para la atención y el mejor resguardo que la persona menor requiere. Madrigal (2009) reseña en su artículo que los albergues donde se atendía a las personas menores de África no encontraban formas de atender situaciones de alimentación, vestimenta y hasta de rituales religiosos distintos a los comunes del país.

18. No se reconoció programas específicos para PммNa en ninguna de las organizaciones consultadas, los cuales fueran dirigidos a informar sobre el conocimiento $y$ la exigibilidad de sus derechos humanos, sensibilizar sobre los riesgos psicosociales que implica migrar sin compañía e involucrarse en una dinámica social y cultural distinta de la suya, así como brindar información sobre los procedimientos que se siguen en el país para la atención de estos casos y las formas de resolución administrativas posibles.

19. Se evidencia la falta de mayor capacitación en el personal que interviene en la atención de la problemática de PммNa, en los distintos momentos del tránsito desde las diferentes instituciones y ongs en el país, a fin de orientar su accionar de un modo sensible con sus particularidades.

\section{Otras consideraciones:}

Se afirma que existe una discriminación etaria al momento de proceder con estas acciones pues hay una prioridad $y$ mayor disposición en la atención de casos de niños y niñas menores de 12 años.
Distintos(as) funcionarios(as) entrevistados(as) coinciden en señalar que en algunos casos la prontitud o lentitud con que se responde en las instancias encargadas de facilitar la repatriación de PMмNa está en relación con la resonancia que el mismo tenga en los medios de comunicación, ya que se presenta una mayor eficiencia cuando es reportado por estos.

\section{BALANCE FINAL}

El interés mostrado por la oгм dirigido a conocer el tratamiento que se da a los casos de pмmna en el país, en el marco de los derechos humanos, llevó al planteamiento de una indagación de tipo evaluativa-formativa para abordar el objeto de estudio.

El proceso para hacer viable el estudio requirió construir un protocolo unificado de actividad a partir de diversos protocolos preexistentes que trataban problemáticas semejantes o asociadas, poniendo en el centro el interés por las pмmna y sus derechos. Este permitiría evaluar cada momento de la intervención interinstitucional de estos casos, desde que la persona menor toma contacto con algún funcionario o funcionaria de alguna institución nacional, hasta que es repatriada a condiciones seguras, o en su defecto, asimilada bajo algún estatus migratorio en el país.

Con este proceder se pueden establecer dos afirmaciones que son el centro de interés de este reporte. La primera es la importancia que tiene la protocolización de los distintos procesos que deben guiar el quehacer de toda institución. En este caso se puede observar que los derechos de las рмммa se ven fragilizados por la falta de protocolización o por el incorrecto ejercicio de lo estatuido.

Las pmmna se mantienen en gran parte del proceso como "migrantes irregulares", ya que debe gestionarse su estatus de refugio; no obstante, no se realiza debido a la falta de coordinación entre la DGME y el pani. Esta realidad debe motivar a establecer de forma breve y clara el modo de realizar esta gestión, así como la obligatoriedad de hacerlo según se le asigne a cada funcionario o funcionaria de una institución. 
Resulta relevante el hecho de que una persona menor queda al margen de ser atendida en centros de salud $y$ de ingresar a algún proceso educativo, hasta que no se haga efectiva su identificación, pues en estas instituciones se requiere el registro de las acciones realizadas en un expediente abierto bajo un nombre verdadero.

Esta problemática se evidencia en mayor medida en las repatriaciones hechas desde las fronteras, tácitamente ilegítimas, sustentadas en la falta de recursos, personal y de la imposibilidad de realizar coordinaciones debido a los diferentes horarios de trabajo entre instituciones. Adicionalmente, por ejemplo, los tiempos en que cada subproceso es realizado o la falta de registros sobre las acciones emprendidas por los funcionarios y las funcionarias de cada institución o sobre las coordinaciones realizadas. Con lo anterior, es claro que si algo no está protocolizado, en ese momento se pueden diluir las expectativas de lograr los objetivos societarios, en el caso de las pmmna, el resguardo de los derechos humanos que les asiste.

El segundo aspecto se trata de la importancia que tiene la investigación evaluativa como recurso para conocer las virtudes $y$ las falencias en el quehacer de toda organización para actuar con fines correctivos, siempre a favor de alcanzar los objetivos que las rige y a los que se deben.

Si se considera que todo quehacer organizativo es siempre perfectible, se resalta entonces la necesidad de conocer los procedimientos o instrumentos que han perdido eficacia con el tiempo, como también los anacronismos que se suscitan y crecen en las organizaciones y en sus coordinaciones, dado los cambios que sufren los ambientes, la realidad social, los recursos tecnológicos, las personas y los objetivos mismos.

En este punto se destaca a la investigación evaluativa como el instrumento clave para producir los conocimientos necesarios y sustentar la toma de decisiones con el fin de alcanzar los objetivos organizacionales. Una organización inteligente debe contemplar la realización periódica de investigaciones evaluativas como forma de mejorar su eficiencia y eficacia, así como, su permanencia en el tiempo.

Un aspecto relevante a tener en consideración es que aunque es factible realizar investigaciones evaluativas en ausencia de protocolos que rijan el quehacer organizacional, tal indagación se tornaría básicamente exploratoria y alcanzaría a producir una información más bien descriptiva. Entonces, la posibilidad de valorar y avanzar en la generación de conocimientos más completos, integrales y explicativos, se logrará en mayor medida si el quehacer organizacional se describe $y$ estatuye ampliamente en protocolos.

Un protocolo es una guía de actividad que se da como producto de una reflexión tanto técnica como ética, sobre la forma de alcanzar los objetivos de la organización en apego a sus valores, visión, misión y metas. Además, como parte del quehacer organizacional estos protocolos deben estar abiertos a sufrir correcciones.

Finalmente, se trata de producir información que permita generar los cambios que las organizaciones requieran. En ese sentido, se concluye que el establecimiento de un protocolo conlleva de forma congénita la realización persistente de indagaciones evaluativas, tal como lo requieren las organizaciones inteligentes.

\section{REFERENCIAS}

Carazo, A. y Salas, S. (2003). La protección de los Derechos fundamentales de los menores migrantes en Costa Rica. (Tesis de Licenciatura en Derecho). Universidad de Costa Rica, Costa Rica.

Chang, H. (2005). A Study of Service Quality, Customer Satisfaction and Loyalty in Taiwanese Leisure Industry. Taiwan: National Kaohsiung First University of Science and Technology.

Chavarría, C. (2014). Necesidades de conocimiento $y$ desarrollo organizacional en la región central de occidente. Meta análisis de las investigaciones realizadas desde los seminarios de realidad nacional. Reflexiones 93 (1), 47-72.

Chavarría, C. y Robert, J. (2011). Situación de niños, niñas y adolescentes que viajan no acompañados por la Región 
Centroamericana. San José, Costa Rica: Organización Internacional de las Migraciones.

Conferencia Regional de las Migraciones (сRм) (2009). Lineamientos regionales para la atención de niños, niñas y adolescentes migrantes no acompañados en casos de repatriación. Guatemala.

Correa, S.; Puerta, A. y Restrepo, B. (2002). Especialización en teoría, métodos y técnicas de investigación social. Investigación Evaluativa. Bogotá: ARFO.

Cruz, D.; López, R. y Ramírez, H. (2007). Organización Inteligente: estudio de caso. Administración y Organizaciones, 10 (19), 101-118. México: uAm. eBSCO PUBLISHING.

Gallardo, H. (1991). Elementos de investigación académica. San José: Euned.

González, C, (1996). Calidad Total. México: McGraw Hill.

González, T.; Moreno, M. y Peris, F. (2001). Gestión de Calidad y Diseño de Organizaciones. Teoría y estudio de casos. Madrid: Prentice Hall.

Hernández, R.; Fernández, C. y Baptista, P. (2003). Metodología de la investigación. Tercera Edición. México: McGraw-Hill.

Madrigal, A. (actualizada el 19 de noviembre de 2009) 8 menores africanos víctimas del 'coyotaje' viven dramática situación. La Nación. Recuperado de http:// www.nacion.com/nacional/africanosvictimas-coyotaje-dramaticasituacion_0_1087091328.html

Martín-Baró, I. (1989). Sistema, grupo y poder. Psicología Social desde Centroamérica II. San Salvador: UcA.
Organización Internacional de las Migraciones (оim)-unicef (2007). Protocolo para la repatriación de niños, niñas $y$ adolescentes víctimas de trata. OIM. San José, Costa Rica.

Patronato Nacional de la Infancia (PANI) (2009). Patronato Nacional de la Infancia. Recuperado el 9 de noviembre de 2009 de http://www.pani.go.cr/spsobrepani.php

Patronato Nacional de la Infancia (PANI)Organización Internacional de las Migraciones (огм) (2007). Protocolo Trata de Personas Menores de Edad. San José, Costa Rica.

Senge, P. (1992). La Quinta Disciplina. México: Granica.

Senile, A. (1996). Calidad Total en los Servicios y en la Administración Pública. Barcelona: Ediciones 2000.

Silverman, L. (1992). Primero estoy yo. El mensaje que sus clientes le transmiten a usted .... Colombia: Norma.

Singh, S. (1999). Control de Calidad Total. Claves, metodologías y Administración para el éxito. México: McGraw Hill.

Venegas, P. (1999). Algunos elementos de investigación. San José, Costa Rica: euned.

Wellington, P. (1995). Cómo brindar un servicio integral al cliente. La mejor de las estrategias kaizen. Colombia: McGraw Hill.

Fecha de ingreso: 16/11/2016 Fecha de aprobación: 05/04/2017 